\title{
COLLIDING WIND X-RAYS IN $\gamma$ VELORUM (WC8+O9I)
}

\author{
ALLAN J. WILLIS \\ Department of Physics and Astronomy, University College London \\ Gower Street, London WC1E 6BT, U.K. \\ HANS SCHILD \\ Institute of Astronomy, ETH-Zentrum, CH-8092 Zürich, Switzerland \\ and \\ IAN R. STEVENS \\ School of Physics and Space Research, University of Birmingham \\ Edgbaston, Birmingham B15 2TT, U.K.
}

\begin{abstract}
Our ROSAT observations of $\gamma$ Velorum obtained over several binary cycles reveal substantial, and repeatable phase-dependent $\mathrm{X}$-ray $(0.1-2.5 \mathrm{keV})$ variations. The derived X-ray light-curve shows a $4 \times$ increase in flux at phase $\sim 0.5$, which is shown to arise in shocked gas formed in the collison of the two stellar winds, viewed through a cavity in the WC8 wind with an observed opening angle of $\sim 50^{\circ}$. This colliding-wind X-ray emission has $L_{\mathrm{x}} \geq 10^{32} \mathrm{erg} \mathrm{s}^{-1}$ and $k T \geq 2 \mathrm{keV}$. Our 2-D hydrodynamical models of the system predict copious $\mathrm{X}$-ray emission from the wind-collision region, at a level and spectrum similar to that observed. Harder (1-10 kev) X-ray observations with the $A S C A$ satellite are being sought to provide improved constraints on the luminosity, spectrum and absorption of the wind-collision emission, and the complex theoretical models involved.
\end{abstract}

Key words: stars: Wolf-Rayet - X-rays - colliding winds

\section{Introduction}

Observations of single OB and WR stars with the Einstein satellite (White \& Long 1986; Pollock 1987) revealed luminosities $L_{\mathrm{X}} \approx 10^{30-33} \mathrm{erg} \mathrm{s}^{-1}$ and soft $(k T \leq 0.5 \mathrm{keV})$ spectra, believed to be produced in the outer stellar winds in shocked gas formed in radiatively-induced instabilities (Lucy \& White 1982; Owocki et al. 1988). In massive binaries, and particularly WR+O systems, the collision of the two stellar winds is expected to produce an additional source of X-ray emission at the interface region (e.g., Stevens et al. 1992). Understanding this colliding-wind phenomena represents a relatively new and challenging field. For most WR stars the Einstein data comprise one or a few observations, and thus provide limited X-ray variability information. In this paper we summarise results from a study of multiple ROSAT Xray observations of $\gamma$ Velorum (WC8+09I). This system has a well-defined orbit, and shows substantial phase-dependent UV P-Cygni profile variability, which St-Louis et al. (1993) conclude provides evidence for a cavity around the 09I star formed by the wind-wind collision, offering the potential for observing $\mathrm{X}$-ray emission produced in the wind collision region. 


\section{ROSAT observations of $\gamma$ Velorum}

Our ROSAT observations were obtained in Guest Observer programmes using the XRT+PSPC (Position Sensitive Proportional Counter), covering 0.1-2.5 keV, imaging over a FOV of about $2^{\circ}$, with a spatial resolution of about 25". During AO2 we secured 10 observations: one on 20 December, 1991 , and a further 9 at roughly 8 -day intervals $(\Delta \phi \sim 0.1)$ during 15 April - 20 June, 1992. These were followed by three TOO observations during April/May, 1993 at binary phases $0.43,0.53$ and 0.61 (all phases use the binary ephemeris given by Moffat et al. (1986), with $P=78^{\mathrm{d}} .5002, T_{\mathrm{o}}=\mathrm{JD}$ 2445768.96, WC8 star in front). The PSPC images show that $\gamma$ Velorum is located in a cluster of 6 , resolved point sources covering about $3^{\prime} \times 5^{\prime}$ of the central FOV. Although $\gamma$ Velorum is usually the brightest source, the other cluster sources combined have a comparable PSPC count, and contribute significantly to the Einstein IPC measurements previously reported (e.g., White \& Long 1986) where the cluster was unresolved. For each observation we examined (i) the total PSPC count-rate and X-ray flux in the 0.1-2.5 $\mathrm{keV}$ range, and (ii) the spectrum and absorption of the X-ray emission. More details are given by Willis et al. $(1994 \mathrm{a}, \mathrm{b})$.

\section{X-ray light-curve of $\gamma$ Velorum}

The measured total PSPC count-rates for $\gamma$ Velorum, are plotted as a function of binary phase in Fig. 1. Also shown is the measured count-rate for one of the other central cluster stars (referred to as Star 3), which shows a relatively constant flux. For $\gamma$ Velorum, our initial AO2 data showed a count-rate decreasing from $\sim 0.15 \mathrm{cts} / \mathrm{s}$ at phase .69 to $\sim 0.07 \mathrm{cts} / \mathrm{s}$ at phase 1.48 , followed by a rapid rise to $\sim 0.42 \mathrm{cts} / \mathrm{s}$ at phase 1.53 ( 0 -star in front). Our TOO observations have confirmed that this effect is repeatable with cycle-phase, and we can therefore fold all the data in phase to produce the X-ray light-curve shown in Fig 1 . Analysis of the PHA-channel data shows that the flux increase at $\phi \sim 0.5$ is entirely due to enhanced emission at hard energies, $\geq 0.5 \mathrm{keV}$, with the count-rates between $0.1-0.5 \mathrm{keV}$ being constant at all phases, implying no significant variation in absorption.

Between $0.0 \leq \phi \leq 0.4$ (O-star remote from WC8 star in line-of-sight), the X-ray flux is relatively constant and at 'low-intensity'. Two-component XSPEC (Shaffer et al. 1991) fitting (black body + photoelectric absorption) to these low-state PSPC data yields: $k T=0.19 \pm 0.04 \mathrm{keV}$, and $N_{\mathrm{H}}=$ $(1.5 \pm 0.8) \times 10^{20} \mathrm{~cm}^{-2}$ (poorly constrained). The latter is consistent with largely interstellar absorption (we estimate $\log N_{\mathrm{H}}($ ism) $\approx 20.0$ from IUE Lyman- $\alpha$ data) and negligible intrinsic wind absorption. Adopting a distance of $0.5 \mathrm{kpc}$, the mean low-state X-ray luminosity derived is $L_{\mathbf{x}}(\mathrm{PSPC})=2.5$ $\times 10^{31} \mathrm{erg} \mathrm{s}^{-1}$, with a range of $[1.2-3.3] \times 10^{31}$, typical for isolated, single WR 


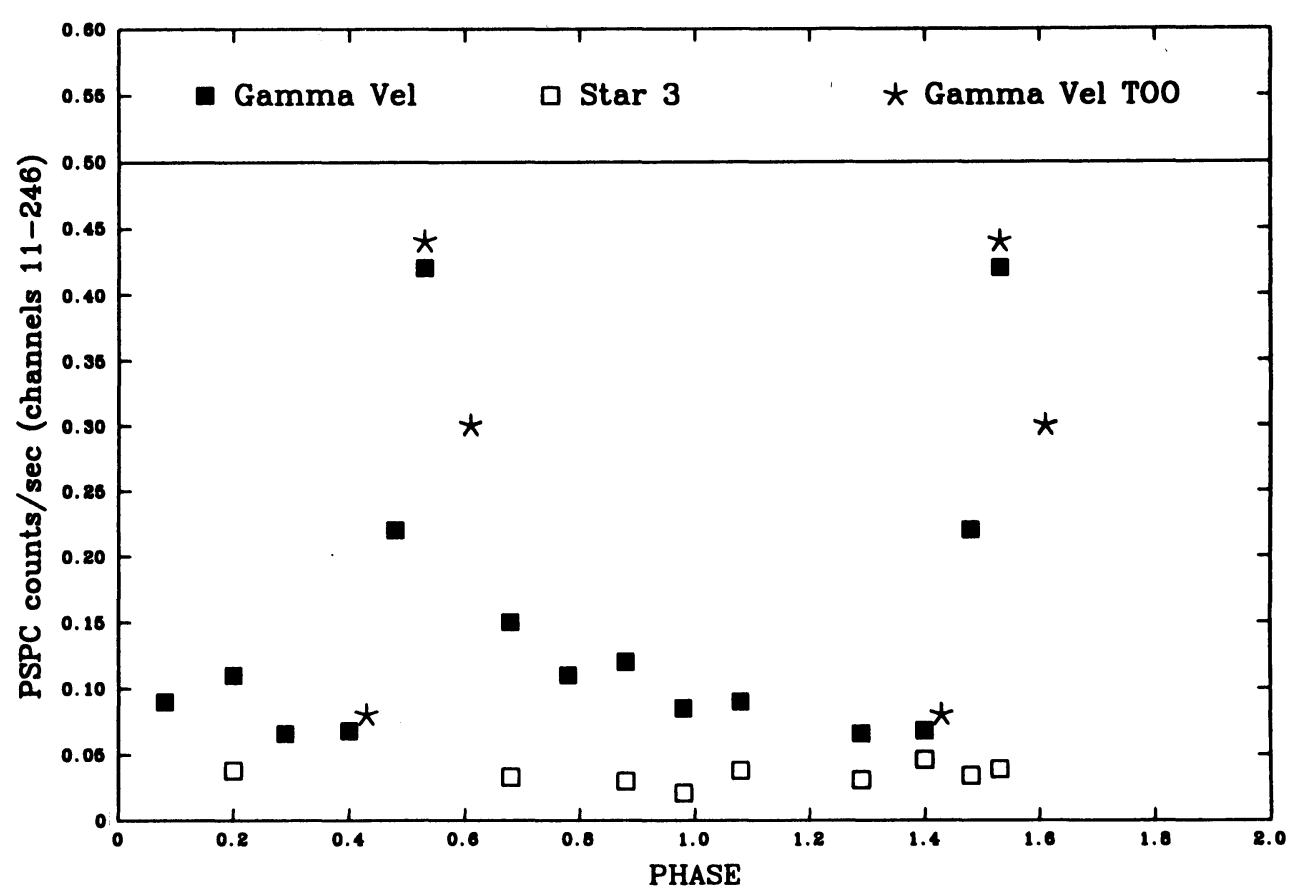

Fig. 1. $\gamma$ Velorum AO2 and TOO PSPC count-rates vs. binary phase

stars. We conclude that this emission arises in radiatively-induced shocked gas in the outer WC8 wind.

The high-state emission from $\gamma$ Velorum $(\times 4$ at phase 0.53$)$ is due to additional, harder X-rays, but the limited PSPC energy range precludes definitive parameterisation. Rough XSPEC fitting, using both black-bodies and Raymond-Smith models, suggest $k T \geq 2 \mathrm{keV}$, and $L_{\mathrm{x}} \geq 10^{32} \mathrm{erg} \mathrm{s}^{-1}$. The phase-repeatability rules out stochastic WC8 wind variations as the origin of this extra emission. Two alternatives are: (i) X-ray emission from the $\mathrm{O}$-star wind, or (ii) X-ray emission from the wind-collision region. In either case the enhanced emission would need to be viewable through a cavity in the WC8 wind formed by the colliding winds. The spectrum $(k T \geq$ $2 \mathrm{keV}$ ) of the enhanced emission is much harder than normally associated with $\mathrm{O}$-star wind $\mathrm{X}$-rays and we believe precludes that origin. We conclude that our derived X-ray light-curve provides the first direct measurement of colliding-wind $\mathrm{X}$-ray emission in this system. From Fig. 1, we deduce an opening angle for the cavity of $\sim 50^{\circ}$. The asymmetry observed in the wind-collision X-ray profile (Fig 1) we believe reflects $\mathbf{O}$-star orbital motion effects 


\section{Hydrodynamical models of $\gamma$ Velorum}

Using the formulation developed for colliding wind binaries by Stevens $e$ t al. (1992) we have computed 2-D hydrodymanical models of $\gamma$ Velorum to calculate the expected X-ray emission from the hot inter-shock gas, including the effects of photoelectric wind absorption, using the binary system parameters given by St-Louis et al. (1993). Detailed results are given by Willis et al. (1994b) and show: (i) a pronounced cavity and high temperature shocked interface region does form around the 0 -star, with a full opening angle of $\sim 40-60^{\circ}$; (ii) at ROSAT energies $(0.1-2.5 \mathrm{keV})$ the emergent X-ray emission from the collision interface is observable only near phase 0.5 , with a predicted $L_{\mathrm{x}} \approx 10^{33} \mathrm{erg} \mathrm{s}^{-1}$; (iii) at harder energies $(2.5-10 \mathrm{keV}$ ) the emergent wind-collision emission is phase-dependent with $L_{x} \approx 6 \cdot 10^{31}-6 \cdot 10^{33}$ $\mathrm{erg} \mathrm{s}^{-1}$ (again peaking at phase 0.5 ). Within current uncertainties in system parameters (e.g., in the mass loss rates and wind velocities at the interface region) these results are fully compatable with our derived ROSAT lightcurve, and confirm our conclusion of a direct observation of wind-collision $\mathrm{X}$-ray emission from this system. Our models show that hard X-ray observations in the $1-10 \mathrm{keV}$ range around phase 0.5 are crucial to determine quantitatively the wind-collision luminosity, spectrum and absorption. We have been allocated GO time for ASCA satellite observations of $\gamma$ Velorum to secure these unique and vital tests and constraints on the complex theory of the colliding wind phenomenon. These ASCA observations should be scheduled for either May or October, 1994.

\section{References}

Lucy, L.B., White, R.L. 1982, ApJ 241, 300

Moffat, A.F.J., Vogt, N., Paquin, G., Lamontagne, R., Barrera, L.H. 1986, AJ 91, 1386

Owocki, S., Castor, J.I., Rybicki, G.R. 1988, ApJ 355, 914

Pollock, A.M.T. 1987, ApJ 320, 283

Shafer, R.A., Haberl, F., Arnaud, K.A., Tennant, A.F. 1991, XSPEC: an X-ray spectral fitting package, ESA TM-09

St-Louis, N., Willis, A.J., Stevens, I.R. 1993, ApJ 415, 298

Stevens, I.R., Blondin, J.M., Pollock, A.M.T. 1992, ApJ 386, 265

White, R.L., Long, K.S. 1986, $A p J 310,882$

Willis, A.J., Schild, H., Howarth, I.D., Stevens, I.R. 1994a, in: A.F.J. Moffat, S.P. Owocki, A.W. Fullerton \& N. St-Louis (eds.), Instability and Variability of Hot-Star Winds (Dordrecht: Kluwer), Ap Space Sci. in press

Willis, A.J., Schild, H., Stevens, I.R. 1994b, $A \& A$ submitted 


\section{DISCUSSION:}

Nussbaumer: To obtain your phase-dependent model you had to include a term for X-ray absorption. How was that done?

Willis: This is done by computing (a) the intrinsic X-ray emission in the colliding-wind interface, as a function of binary phase, and (b) computing the photoelectric absorption in the sight line through the $\mathrm{O}$ and/or WR wind, using solar abundances for the $\mathrm{O}$-wind material, and WC abundances for the WC8 component. Full details are described by Stevens in his talk this afternoon.

Koenigsberger: Given what you are seeing in $\gamma$ Vel and simulations such as Ian Stevens is calculating, would you expect to see the X-ray modulation if the orbital separation were much smaller (c.f. 50-60 $\mathrm{R}_{\mathrm{o}}$ )?

Willis: I have no idea - that is something for the modeller/theorists to determine. The other thing, as an observer, is to go and look with a phase-dependent X-ray programme, of the kind we have done for $\gamma$ Velorum.

Meurs: As a technical comment, one additional reason to encounter some problems with spectral fitting is that the observed data plus fits that you showed contained more data points than would be justified in view of the spectral resolution of the ROSAT PSPC.

Willis: I am, of course, aware of that. In our PSPC spectral fitting with XSPEC, we first bin the individual PHA channel data to ensure that each bin contains a large number of counts. So there is no problem in our spectral fitting, where typically we have over 1000 counts in the individual pointing datasets.

Cherepashchuk: Do you have some evidences about rapid variability of X-ray at timescales $\sim 1000 \mathrm{sec}$ ?

Willis: In our high-state emission of phase 0.5 (in both the $\mathrm{AO} 2$ and $\mathrm{TOO}$ datasets) there is some evidence for variability within the individual ROSAT pointing data. This may reflect the turbulent-instability strucrures that the 2-D hydrodynamic codes predict. There is no evidence for any short-term variability in the low-state (WC8-wind) X-ray emission. 